\title{
The Psychology of Online Sociability
}

\section{Theory and Examples}

\author{
Clemmensen, Torkil
}

Document Version

Final published version

Publication date:

2006

\section{License \\ CC BY-NC-ND}

Citation for published version (APA):

Clemmensen, T. (2006). The Psychology of Online Sociability: Theory and Examples.

Link to publication in CBS Research Portal

\section{General rights}

Copyright and moral rights for the publications made accessible in the public portal are retained by the authors and/or other copyright owners and it is a condition of accessing publications that users recognise and abide by the legal requirements associated with these rights.

\section{Take down policy}

If you believe that this document breaches copyright please contact us (research.lib@cbs.dk) providing details, and we will remove access to the work immediately and investigate your claim. 


\section{THE PSYCHOLOGY OF ONLINE SOCIABILITY: THEORY AND EXAMPLES}

by

Torkil Clemmensen

Copenhagen Business School HANDELSHØJSKOLEN
Department of Informatics Howitzvej 60 DK - 2000 Frederiksberg 


\title{
THE PSYCHOLOGY OF ONLINE SOCIABILITY: THEORY AND EXAMPLES
}

\author{
Torkil Clemmensen \\ Department of Informatics \\ Copenhagen Business School \\ Howitzvej 60, 2000 Frederiksberg \\ Denmark \\ Phone +4538152389 \\ Fax +4538152401 \\ Email: tc.inf@cbs.dk
}

\begin{abstract}
In this chapter, I will review current approaches to online sociability and present and exemplify a psychological theory, the Social Reality theory, of online sociability. By analyzing sociability in a virtual world based university course, I will present and analyze examples on how to understand the students' design of the conditions for sociability as communication about cultural symbols, such as avatars and virtual landscapes, and the social reality of perceived groups of people. The analysis results will be used to illustrate different kinds of online sociability: superficial, convivial, and negative sociability. The chapter suggests solutions and recommendations to designers and researchers with a focus on online communities and networked communication.
\end{abstract}




\section{INTRODUCTION}

This chapter deals with the design of sociability for human encounters in online environments. By analyzing sociability in a virtual world based university course, I will provide a psychological perspective on users' design and practice of online sociability. I will present and analyze examples on how the students' design of bodily aspects of the virtual world, e.g. the students' choice between the limited and to the purpose insufficient number of different avatars, contributes to shape the roles and rules of their social interaction. In the context of basic, everyday classroom activities, students' virtual world social interaction and communication take unexpected and interesting forms. For example, in the virtual world based university course that I have analyzed here, one form of student interaction resembled the famous Wild West gunfight between the gangs at the OK Corral in Tombstone. Such unexpected projections in time and place of groups of people will be psychologically real and unpleasant to the participants who are surprised by the scenarios, in which they suddenly find themselves embedded. Furthermore, such odd forms of online class social interaction are difficult to understand if we only look at the communication from a mass media perspective or from an interface design perspective. We need to find a concept that bridges these two areas. A useful bridging concept between Computer Mediated Communication and Human Computer Interaction is the concept of online 'sociability', which has been investigated from sociological, technological and mass communication perspectives (Preece, 2000). In this chapter, I wish to add to the knowledge of online sociability by presenting a psychological perspective on sociability to support the analysis, design and evaluation of online communities and networked communication.

\section{Background}

From the early days of the information and communication technology field, the concept of sociability has been important to practitioners and researchers. For example (Willoughby, 1972) described the individual software programmer's personal ability to socially interact with other people as a sociability issue, and (Mantei, 1981) discussed the sociability requirements to small teams of computer consultants who should be able to speak to users as well as to technical specialists. This early psychological view of 
sociability within information and communication technology environments needs to be updated in the light of recent technological and theoretical advances.

With the emergence of the internet, the concept of sociability has gained a renewed interest within information and communication technology design as a way to express and study (mostly negative) social aspects of the technology (Kiesler, 1998). Studies show that users do not become more sociable by the new networked communication possibilities offered to them by the internet, and that the time internet users spend online actually reduces interpersonal interaction and communication in much the same way as watching TV does (Nie, 2001; Nie \& Hillygus, 2002). It is a fundamental mistake, it is argued, to think that the role of networked communication is to maintain or develop spatially remote social ties; we will not get new friends just because we get access to the internet, and most of the social ties that we maintain by telephone are those that are based on blood relations (Gourna \& Smoreda, 2003).

There are, however, positive social effects of participating in dedicated social activities on the internet, i.e. activities on the internet done with the purpose of having social interaction in cases, when the alternative is little or none social interaction. Today much of the (inter-)networked communication takes place within online communities of various kinds: communities of practice, communities of interest, communities or learning, health support communities, etc. New technology for networked communication seems to work as a principle that organize the social ties where urban mechanisms do not work (in big cities, large buildings, institutions and companies, etc) and give new possibilities for convivial proximity requiring coordination of time among family members and friends (meeting friends in town, women’s’ night work, etc.) (Gourna \& Smoreda, 2003). However, to plan and design for successful networked communication in emergent online communities requires more than just the design of the human-computer interaction (usability); it requires planning for sociability (de Souza \& Preece, 2004).

Planning for sociability in online communities is a well investigated area of research in recent years. We now have knowledge of how sociability is related to networked 
communication in terms of the success of online communities. The success of an online community can be measured in terms of the numbers of participating people, the number and interactivity of the discussions, and the kind of policies in place (Preece, 2001). Relevant sociability measures of success include classification and counts of uncivil communication behavior such as abusive language, and issues of trustworthiness such as the adherence to procedures for making the trustworthiness of e-commerce action vendors explicit. Other sociability measures of success include counting the amount of 'lurkers', i.e. people who read or see communication content, but who do not contribute themselves with messages, and counting the amount of on-topic conversations and reciprocity in giving and taking questions and answers in discussion threads, etc. (Preece, 2001). Further development of measures of environments designed specifically for enhancing social interaction may be based on validated instruments such as social space scale, social presence scale and sociability scales (Kreijns et al., 2004). The promise is that we soon have valid and reliable measures of sociability.

Measures of sociability need however to be interpreted within theoretical frameworks. Currently a semiotic and sociological framework can be used to support evaluators, designers, moderators and users in their identification and understanding of sociability problems (de Souza \& Preece, 2004). The idea is that online communities have three constituents: 1) an online community constituent with four ontological elements: community, people, purposes and policies, 2) an interpretative constituent that provides test of the adequacy of the communication behavior in the community by applying a base of culturally determined signs systems (signs given in the semiotic sense, such as common conversational signs familiar to any speaker of a given language), together with the 3) third constituent of the online community framework: the usability and sociability constituent that examine both individual attributes such as thrust and reciprocity and collective attributes of sociability such as the culture of a community (de Souza \& Preece, 2004). This framework can then be used to analyze particular instantiations of the general online community concept. For example, it has been used to analyze an education support community and suggest changes in the design of the community's software (de 
Souza \& Preece, 2004). Further research related to this framework may include framing it within other theory, for example within psychological theory (de Souza \& Preece, 2004).

Empirical studies, in particular ethnographic research into online sociability on the internet and in virtual worlds, have helped unveil sociability phenomena of networked communication. To a degree, these online phenomena in all their complexity and unpredictably are similar to phenomena known from sociability in offline settings. For example, the offline sociability emerging in American-Israeli family dinner conversations (Blum-Kulka, 1997) resembles the complex composition of roles and behaviors that may emerge in course of the communication behavior in online communities, even in an online community with an undifferentiated technology for support of sociability (Maloney-Krichmar \& Preece, 2005). Such emergence of complex behavior makes externally-driven governance of the community unnecessary, because newcomers learn to behave simply by deducing suitable roles (e.g. task roles, socio-emotional roles, individualistic roles) from reading existing threads in the community and following the norms these roles suggest (Maloney-Krichmar \& Preece, 2005). Another example of the complexity and unpredictably of online sociability is the finding that contrary to early predictions of the effect of internet usage (Kiesler, 1998) people actually benefit socially from participating in online communities; they find emotional support, information, and esteem support as well as humor in the exchanges among community members (Braithwaite et al in (Maloney-Krichmar \& Preece, 2005)). A third example of the complexity of online sociability and its comparability to offline sociability is that studies of online communication acts in the home tells us that people plan for personal conversations, they plan time and place and even place a telephone call to the other person to arrange the best time for the personal exchange; and, furthermore, besides this specialization of space there also seems to be a homophily of contacts, i.e. people communicate mostly online with people they know of same sex and are actually quite inaccessible to other possible communication partners (Gourna \& Smoreda, 2003). A fourth example of the complexity of online sociability is the identification of dedicated places for sociability in massive multiplayer games, which shows that online 'cantinas' like offline cantinas helps facilitate the long periods of inactivity that may be a necessary 
condition for sociability to occur; the lessons learned here is that it is important for the development of sociability to promote regularity, keep in the most active socializers among the community members, and to partition the social space by making separate rooms in the cantina. A fifth example of the complexity of online sociability is the (positive and negative) evident changes of the thinking and values of first time participants in an (online) community that by design allows for culturally non-accepted behavior, despite the participants' intention to use the communication medium in accordance with their original cultural values (Al-Saggaf \& Weckert, 2004). A sixth example of the complexity of online sociability is the lower sociability threshold (do not talk about personal trouble) and an upper sociability threshold (do not discuss general differences among people) that support newsgroup participants' sociability in the original playful sociological sense of the word, see (Simmel \& Hughes, 1949), (Rutter \& Smith, 1999). These examples of ethnographic research of online social interaction suggest to me that rather than inhibiting inappropriate behavior by using rules and policies in the support of emerging online communities, it may be better to focus on supporting the development of strong group norms through moderation and reinforcement of positive group behavior and to partition the conversational space by a good design. Having such a focus underscores the need for a psychological study of group perception and the role of communication in sociability.

Technology studies of social support technology illustrate various possibilities for designing for sociability. There have been attempts to develop technology with 'social affordances'; i.e. technology with properties that facilitate social context by for example providing salient information to all about users and enables communication with other users (Kreijns et al., 2002). A concrete attempt to develop such a technology is the design of personal devices with built-in 'social functionality', i.e. those aspects of a device that is explicitly designed for eliciting a sociability (Constas \& Papadopoulos, 2001). This sociability doesn't even need to be an explicit goal for a user of the product, but is designed for just coming to a user's mind when he or she uses the device. An example is the design of a bracelet that allows the user to withdraw money from an ATM as well as functioning as a jewelry, and in the latter function increases the enjoyment of being 
together with others (Constas \& Papadopoulos, 2001). Another example of a technology appropriating social affordances is how online shopping can be supported by developing shared browsers that is designed with the intention to make people enjoy developing common ground while doing online shopping together (Farnham, 2001) and by adhering to usability and sociability principles in the design of online customer service chat (Andrews \& Haworth, 2002). Similarly, the sociability of computer supported collaborative learning can be supported by social contextual facilitators like a group awareness widget that provides a learner group with the awareness about the other members of the group, and, furthermore, provides the learner group with a particular focus on off-task interactions of central importance to the social psychological experience of sociability (Kreijns et al., 2002). Communities of science teachers can be supported by a web based tool designed around the 'visiting-the-classroom' metaphor with an aim of fostering sustained participation and a sense of ownership (Barab et al., 2001). Students in classrooms can be supported by software that allows them to be co-constructors of their own learning environment so that it appears fun and sociable to them (Ryberg \& Ponti, 2004). Summing up, technology studies identify possibilities for design technology that supports social interaction, and point to the importance of technology that allows the human user an active role in the design of online sociability.

Thus we have a sociological concept of sociability of online communities and a semiotic framework for analyzing and understanding sociability in communities, and we have measures for evaluating online communities. We have ethnographic accounts of online sociability phenomena, and we have technological solutions to a number of sociability problems in a diverse set of environments. The rest of this chapter tries to give an updated psychological concept of sociability that by looking at individuals and small groups' social skills and knowledge takes up the thread from the early understandings of sociability within the information and communication field.

\section{A Social Reality theory of online sociability}

In this section I present a Social Reality theory of online sociability based on a discussion of the role of psychological essentialism and group perception in online sociability. 
Group perception, or the social ontology of groups, concerns how to determine whether an aggregate of individuals is an entity or not. From a psychological viewpoint this process is a human capacity for visually analyzing very rapidly if we confront a grouplike entity, i.e. if a number of individuals really are a group in our eyes. In order to rapidly and visually experience groups of people, we use gestalt principles such as proximity, similarity and common fate, in much the same way as we use gestalt principles to perceive groups of objects (Campbell, 1958).

Having a psychological sense of the reality of a social group is strengthened by ongoing mutual verification of collective intentionality: We all the time confirm each other in the group that we aim at a shared target. How do we do that? Symbols are cultural, they are entities based on implicit or explicit social agreements. However, they are also used to create such social agreements. In other words, cultural symbols and social agreements are locked to each other in a seamless process that verifies our collective intentionality. In this process, human language communication plays a major role as the activity that binds culture and social reality together (Kashima, 2004).

Culture supplies shared symbols that enable people to construct their social reality. Social reality then underpins the cultural symbols which helped to construct the social reality to begin with, and it is the communicative act that gets this process going. This cycle creates the psychological sense of social reality. For example, an Indian student may discuss with me as a visiting researcher a drawing of Ganesh, the Hindu elephant god: the nature of this drawing (new to me, familiar to the student, etc.) creates a possibility for a social reality with me as a guest and the student as a representative for the host; this possible social relation is underscored and supported by our communication that reveals that the student think of me and my knowledge as being on the level of a tourist and thus different and lower that any of the other Indian students' and professors' knowledge of Ganesh; from then on our communication about the Ganesh symbol and our little, dyadic social reality will maintain and perhaps develop this newly created social reality. For a visual illustration of the process, see Figure 1, which is adapted and developed from the figure in (Kashima, 2004, p263) in order to focus on the production of various forms of online 
sociability. Figure 1 illustrates the triadic relationship between cultural symbols (such as the Ganesh symbol in the example above), social reality (in networked communication enabled by the technologies of Human-Computer Interaction (HCI) \& Computer Supported Cooperative Work (CSCW)) and communication processes (which in networked communication is synonymous with Computer Mediated Communication (CMC)). The placement in the model of online Sociability between social reality and communication indicates how online sociability emerges both from computer mediated communication between humans, and from individual and cooperative computer supported interaction with environments. Figure 1 also illustrates how these processes may result in various forms of online sociability.
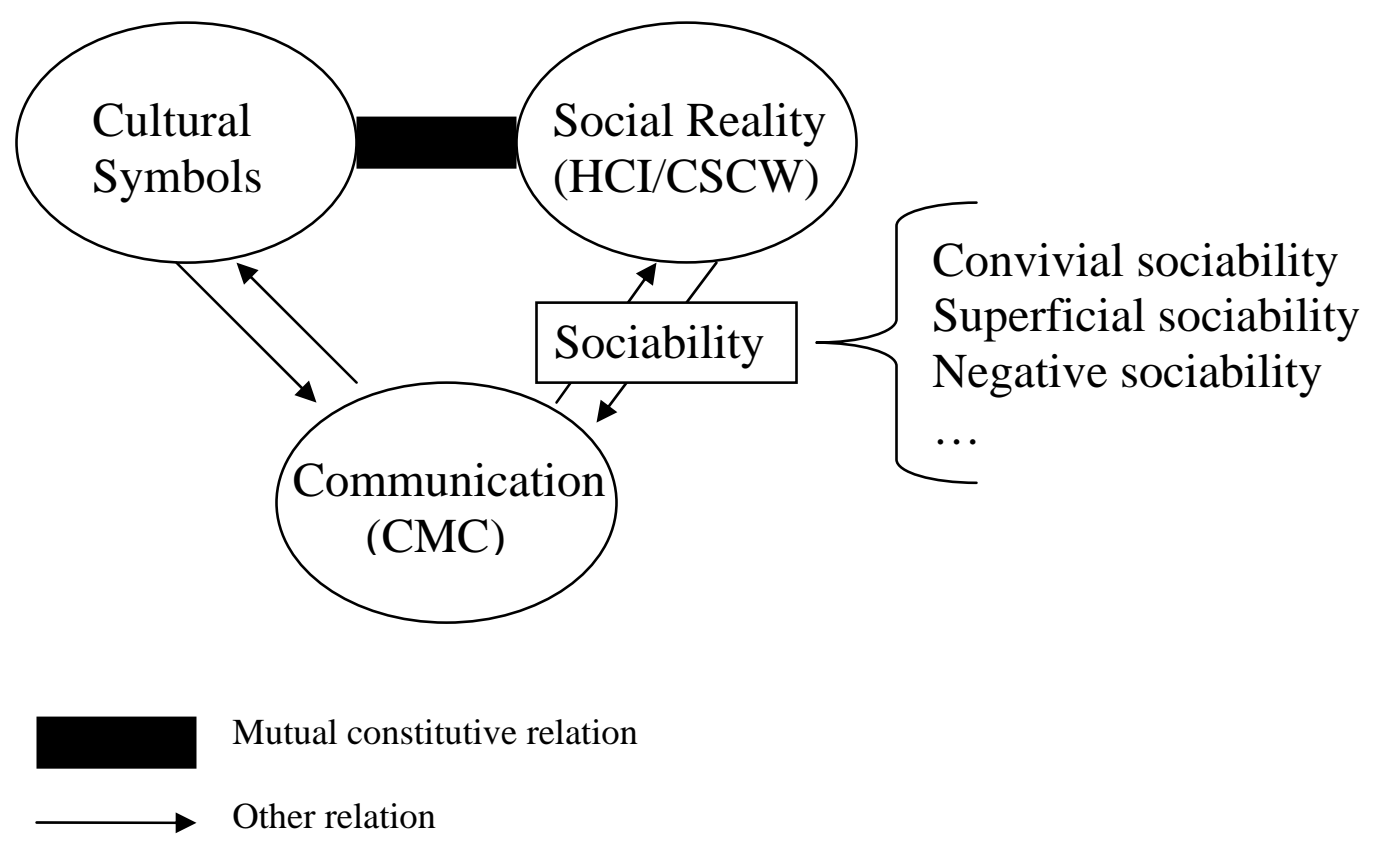

Figure 1. The production of various forms of Online Sociability

I call my adaptation as it is illustrated in Figure 1 of the psychological group perception theory to online sociability issues, the 'Social Reality theory of Online Sociability'. With the theory, I suggest that we think of sociability as the psychological sense of social reality. Measuring sociability then means measuring the psychological sense of social reality, which is not a component of a theoretical framework but rather an emerging, dynamic phenomena; being sociable means displaying a good personal sense of social 
reality, and designing for support of sociability means designing support for the psychological sense of reality. If we take into account that cultural symbols are mutual constitutive with HCI/CSCW realities, sociability then becomes a bridge between CMC and $\mathrm{HCI} / \mathrm{CSCW}$ in various ways.

Social Reality theory of sociability as illustrated in Figure 1 focuses on the human experience of social reality. This makes the theory different from other attempts to formulate a social psychological theory of online sociability. For example, the theory of social affordance and sociability of computer supported cooperative learning environments (Kreijns et al., 2002) define sociability as the extent to which the computer supported cooperative learning environment give rise to a social space. Comparing this theory with social reality model of sociability, the computer supported cooperative learning environment including the social affordances may be understood as a repository of cultural symbols, and the social space as a kind of social reality that emerge from communication in media that allows and supports social presence. However, this affordance based concept of sociability is not a psychological one, but an attribute of the environment only: "Sociability is a strength or potential associated with an environment", (Kreijns et al., 2002) page 10). While social affordance theory thus holds that sociability is a quality of an environment, Social Reality theory maintains that sociability is a human experience.

The position advanced in this chapter is that sociability in a psychological sense depends on specific cultural symbols, specific social reality(ies), and the concrete communication processes that develop and maintain the relations between these. As researchers, we are part of this communication. Therefore, depending on which of the relations in the Social reality model (arrows in Figure 1) that we choose to investigate, we will get a certain view of online sociability, and, reversely, if we investigate particular phenomena of social reality, then this will focus our eyes on particular relations in the model.

When a group becomes a part of social reality, people develop a sense of the group's existence being out of their control or manipulation; this is psychological essentialism 
(Kashima, 2004). In other words, the existence of a particular group with (a) perceived similarity in members' goal directed activity, (b) perceived member characteristics such as appearance and character, and (c) perceived physical location, may gradually become an implicit assumption in peoples’ everyday reasoning. Then it becomes very hard for the group and group members to alter the group’s characteristics (Kashima, 2004). For online sociability, psychological essentialism means that groups seemingly exist outside human control or manipulation by HCI/CSCW design or CMC activities, and that any online group may become just as real and hard to alter as any offline group.

\section{THE SOCIAL REALITY THEORY OF ONLINE SOCIABILITY ILLUSTRATED}

In this section, I apply and illustrate the Social Reality theory of online Sociability on issues of unexpected and seemingly odd social interactions in a virtual world university course I gave on computer supported cooperative work and online communities. I present my perspective on the forms of interaction and communication that emerge in networked communication, and relate these to the thesis of sociability as a bridge between HCI/CSCW and CMC, and to my position that this bridge can only be built if we include cultural symbols in the model of social reality. Furthermore, the analysis I present shows that users are active in designing their own sociability experiences. They are, however, not successful in all cases, and therefore it is important to consider what we as designers and researchers can do to help users’ improve their sociability experiences.

In the following I draw material from a virtual world (Active world) session that I held with my graduate students at a course on CSCW and online communities. At this session, they were supposed to present their experiments with on- and offline small-groupcooperation about a task (Towers of Hanoi). Their presentations should be done in the Active World online environment during a two-hour long sessions in which we all were physically located either in our home or at diverse computers at the university campus. As part of the setup, I did a post-session evaluation the week after when all the students met face-to-face with me in the classroom. In this evaluation, I interviewed and moderated the students telling and discussion about their experiences during the online 
session, while I showed them all the screen dumps that are presented and discussed in the following.

First I discuss the 'Meeting at the OK Corral', in which three male students took the position of a group of uniform gun-slingers confronting an unidentified enemy (see the figure below). I introduce the non-verbal, implicit, silent, gender-specific, media-oriented and cultural thinking that these young men revealed, when I asked them in the classroom after the session why they chose their appearance as they did. Second, I analyze how the students moved from presentation to presentation in the virtual world and reflect on why this very simple problem of moving together in a virtual world is considerable harder to solve than it at first appears to be. Third, I report from an event, which was afterwards characterized as sexual harassment from an unknown student's avatar by the female student who was the victim of the other's avatar's closeness. Then I present the observations on students' implicit rules and roles of interactions in a case where one group had chosen to do their presentation high above the ground in the virtual world. Finally, I report on how the students presented their results as facts in their poster sessions using unusually large poster formats. Together these forms of interaction and communication can be explained by different aspects of sociability, but not without considering the context provided by the students’ activation of highly diverse and sometimes misplaced cultural symbols.

\section{Superficial sociability: Meeting at the OK corral}

An important point in the Social Reality theory of Sociability presented in figure 1 is how cultural symbols and HCI/CSCW-enabled social reality are mutual referents and together form a basis for experiencing a form of sociability in the CMC communication. This point will be illustrated by an analysis of an odd sociability experience related to the beginning of the course session, when students had come together to watch the first group present their work, see Figure 2. The example illustrates and introduces what I call superficial sociability. 


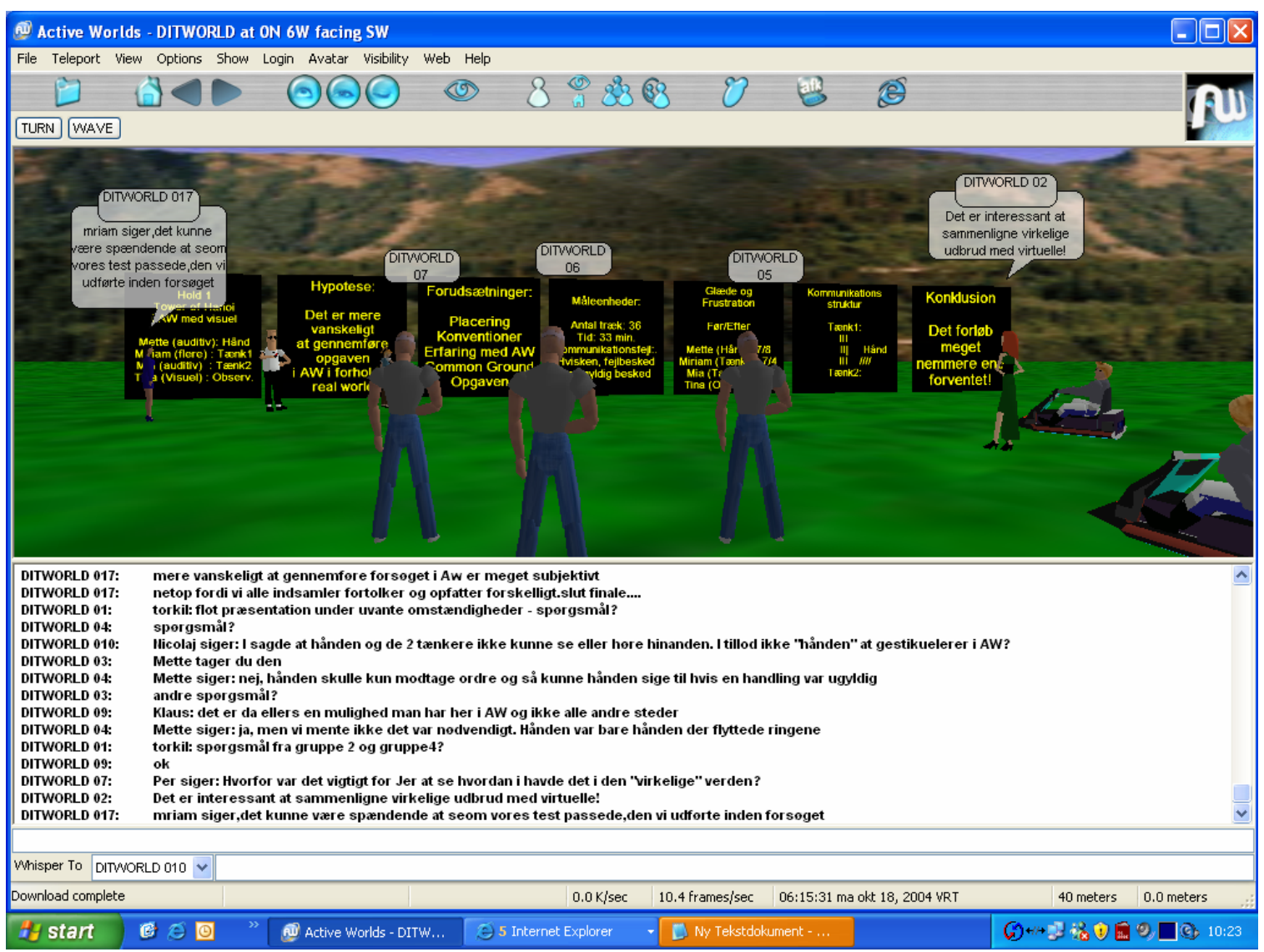

Figure 2. Meeting at the OK Corral - An illustration of superficial sociability.

In the situation shown in the figure, three male students take the position of a group of uniformed gun-slingers confronting an unidentified enemy, much like the standard configuration on the street of a small western town in a cowboy movie. The three male avatars are at same physical location and are similar in appearance, and the other participants in the virtual world session rapidly perceive them as a uniform group. Furthermore, their group activates the cultural symbol of Wild West gunslingers, due to their 'spread out' position, their 'strong men' appearance, their similar names with subsequent numbering, and the lack of visual cues to their purpose other than surrounding wild nature. Had they and other students, instead of being surrounded by Wild West nature with mountains etc, been sitting on rows of chairs we would have known that they shared the purpose of listening to a student presentation. None of this ambivalent reality, 'Wild West social reality' vs. 'listening student reality', is communicated in the online chat text which only concerns the ongoing presentation and questions and answers related 
to that. In the post-virtual world session discussion in the class about their 'Wild West appearance', however, the three students explained to the other students that they selected three similar avatars in order to appear as a group and that their 'spread out' location actually was in order to allow everybody better to see the presentation. They had not attempted to design their shared appearance at the presentation as a 'threatening group' of gunslingers as some of the participants said they were, but had on the other hand neither considered how they should look like if they should to appear as 'listeners' to the other participants in the student presentation.

It is clear that the three students’ psychological sense of social reality, their sociability, was only superficial. Their sociability was based on an apparent similarity in appearance and location, but lacked a clear common fate. Furthermore, it suffered from the wrong cultural symbols, i.e. it suffered from the 'wild west nature' surrounding in the $\mathrm{HCI} / \mathrm{CSCW}$ environment of the 'Active world' and from the lack of a 'listener' avatar or other cultural symbols which could be useful in the construction of a suitable social reality. Obviously the students were not supported or encouraged to communicate about this during the session, for example by being asked if they were a group, and if yes, what kind of group they were. Hence the Wild West cultural symbols in the HCI/CSCWenabled social reality formed via the CMC communication a basis for experiencing a superficial sociability. The students in the example presented here by their own explicit design, and with very simple means of choosing similarity in appearance and physical location, managed to stand out as group.

In contrast to the students accomplishment, we know from previous work that so-called ‘third places', i.e. online places dedicated to online sociability in particular, but also online environments in general, tend to erase individual distinctions between participants (Ducheneaut et al., 2004). Intuitively this generalized experience seems always true, because usually everybody in virtual worlds only has a limited number of avatars to choose between and they can do more or less similar things. In contrast to this intuition, however, the three gunslinger students in the Wild West example presented above successfully designed and communicated a superficial sociability that made them as a 
group stand out from the other student groups. By supporting the production of this particular superficial sociability, they actually counteracted a potential leveling out all individual distinctions between participants.

The students, however, by their design of their appearance as a group gained something they had not designed for: they appeared to be a threatening group. The phenomena of importing forms of social interaction from outside the online social reality, is well known - and warned against - in research on sociability in computer supported cooperative learning (Kreijns et al., 2002). The OK Corral situation is an example of the importance of the social psychological dimension of social interaction outside the task context and how the (non-)communication about cultural symbols affects online sociability.

\section{Convivial sociability: Moving collectively}

According to the Social Reality theory of Sociability, the CMC communication facilitates participants' psychological sense of social reality and thereby partly constructs their social reality and their cultural symbols. This point will be illustrated by an analysis of an odd sociability experience appearing in the course session at the time when students just finished their first group presentation and now needed to move to another place in the virtual world in order to watch the next student group present their work (see Figure 3). As should be clear below, the students experience convivial sociability, which I attempt to exemplify by pointing out that the students' extensive communication allows them to experience having a common fate, without having to superficially appear similar or to be proximal in their location. 


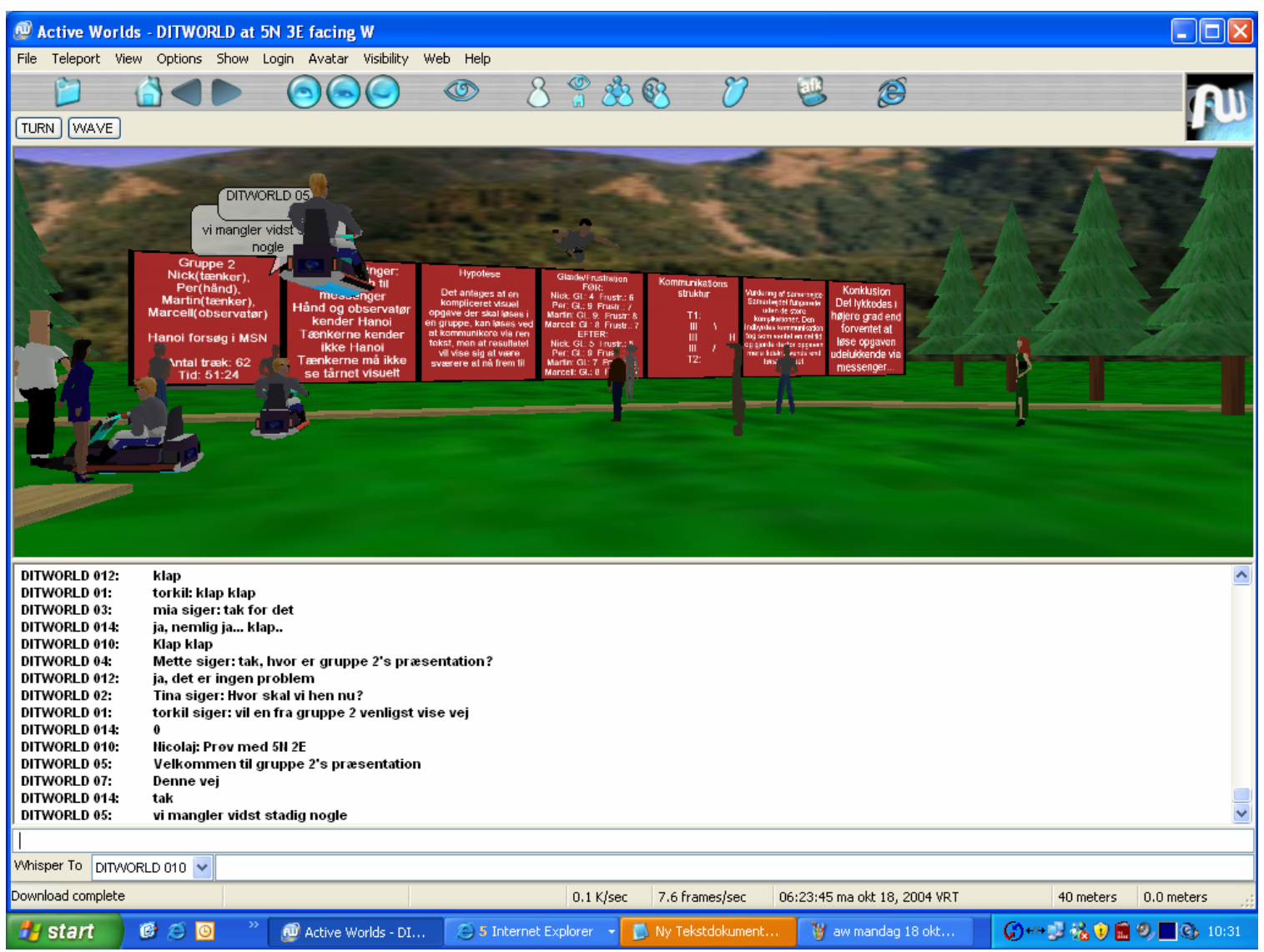

Figure 3. The communication and the social situation after the students and their teacher have moved collectively to a new presentation site. The situation illustrates convivial sociability.

In the excerpt from the chat communication shown below (and in Danish in Figure 3) two students ask where everybody have to go now to watch the next presentation, and people begin to move away from the location in a scattered way. The teacher asks out in the blue for directions and a student from the presenting group gives geographical coordinates (see the following translation of this part of the communication in Figure 3):

DITWORLD 04: Mette says: where are group 2's presentation? DITWORLD 012: Tina says: where are we going now?

DITWORLD 01: Torkil (teacher) says: Will somebody from group 2 please show the way?

DITWORLD 010: Nicolaj says: try SH 2E

DITWORLD 05: welcome to group 2's presentation

DITWORLD 07: this way 
DITWORLD 014: thank you

DITWORLD 05: we are apparently still missing somebody

Without warning and in a in a glimpse of time we are in the social reality illustrated by Figure 3; the information from Nicolai appeared to be an address in the virtual world which made it possible for participants to immediately move from the location of the first group presentation to the location of the next group presentation. In this new location, two of the presenting group members (DITWORLD 05 and DITWORLD 07) say welcome. While on their way they have changed their avatars from the previous 'gunslinger' avatar to 'motorcycle' avatars. In the middle of the picture we also see an avatar standing on its head. In other words, there are several quite different avatars present, and still have not all the students arrived at the site yet - those who cannot figure out how to use the software to teleport themselves to the presentation site are 'out', i.e. they are left behind.

In the example, the student's limited and misplaced (out-of-class-room) vocabulary in the computer mediated communication, the avatar-changing class-mates, and the social reality of student presentation, combine into an odd sociability experience. It is odd to be as a student or a teacher in a world with a blue sky, having to find the way by using geographical coordinates instead of class room numbers, and some of the students suddenly becoming motorcyclists: Who would ever, in an off line class of students, ask a student on a motorcycle to present at, say, 56 degrees north, 10 degrees east? However, the students seem happy, positive and polite towards each other: they say 'this way' and 'thank you' and they care for others 'we are apparently still missing somebody', and they seem to be able to in their communication to make clear that they are a student group. The students seem to experience the collective moving around as emotionally positive and rewarding in this particular case.

In research on the rituals of sociability in CMC in health communities, it has been found that the enactment potential of 'netiquette' is closely associated with the production of at least five features of positive, convivial sociability: 'discussion thread' organization and content (standard discussion thread lengths will be borne out of participants every day 
experience in discussions), terms and techniques of address (recognizing who is being addressed etc), broadcast requests (such request are being met with advice and response to advice), playful re-contextualization (to find new twists and turns in the development of a thread) and sociability thresholds (not to be too personal and report on own divorce troubles etc, and not to discuss political and religious issues) (Rutter \& Smith, 1999). If we compare these research findings on the production of convivial sociability in health communities with the example presented in Figure 3, it is possible to see the rituals of sociability in the virtual world university course and how they relate to communication: Firstly, the length of the statements seems to be shared by the students. Secondly, they make it clear who speaks by entering their real name as the first word in the statement which is a very fast ritualizing of communicative behavior, since they have only been online together for the first time in their life in less than half an hour. Thirdly, advice is given in terms of addresses and directions and the advice is acknowledged by the

receiving students. Fourthly, the neutral addressing of the missing students (no names on fellow students, and no positive or negative generalization of the missing students) in the example in Figure 3 can be seen as an example of sociability thresholds in action.

A theoretical understanding of convivial sociability will help define online sociability qualitatively, in contrast to definitions of degrees or extent of sociability (Kreijns et al., 2004). Convivence, 'living together' in the working environment, in organizations, is a human experience that should not end up in any degree of suffering and human intolerance (Avallone et al., 2005). In contrast to the current theoretical interpretation of the enactment of netiquette in online communities, which merely suggests that an appropriate netiquette leads to 'discourses of friendliness', see (Rutter \& Smith, 1999), the Social Reality theory of Online Sociability offers an interpretation that is rooted in an understanding of the importance of communicating about cultural symbols and social reality in order to produce online sociability.

\section{Negative (violent) sociability: What's going on?}

The part of the psychological model of sociability in figure 1, which I illustrate with the next example concerns how the nature of the available cultural symbols hinders a 
humanlike CMC communication. Hereby a stiff social reality is created, which is experienced as odd and to some even a violent sociability.

The (odd) sociability experience that I present is when students are in the midst of their second group presentation, see Figure 4. In the left of the picture, one of the students has changed into an airship avatar which hovers over the ground. In the mid of the picture, the student who uses a male motorcycle avatar DITWORLD 012 runs his avatar into the female avatar DITWORLD 017, who does not react to being run into. Otherwise the presentation is proceeding, as is evident from the written communication. However, when asked next time the class met face-to-face, the student whose avatar had been run into expressed that she (it was a female student) had felt it unpleasant on obtrusive to be hit by the motorcycle avatar, but she didn't know what to do about it. When the female student one month later delivered her term report, she had written about the event and her experience of being run down several times, but luckily survived. When she two months later got a chance to speak about this again, at the individual oral exam table, she voluntarily returned to the event and spoke about is as molestation. 


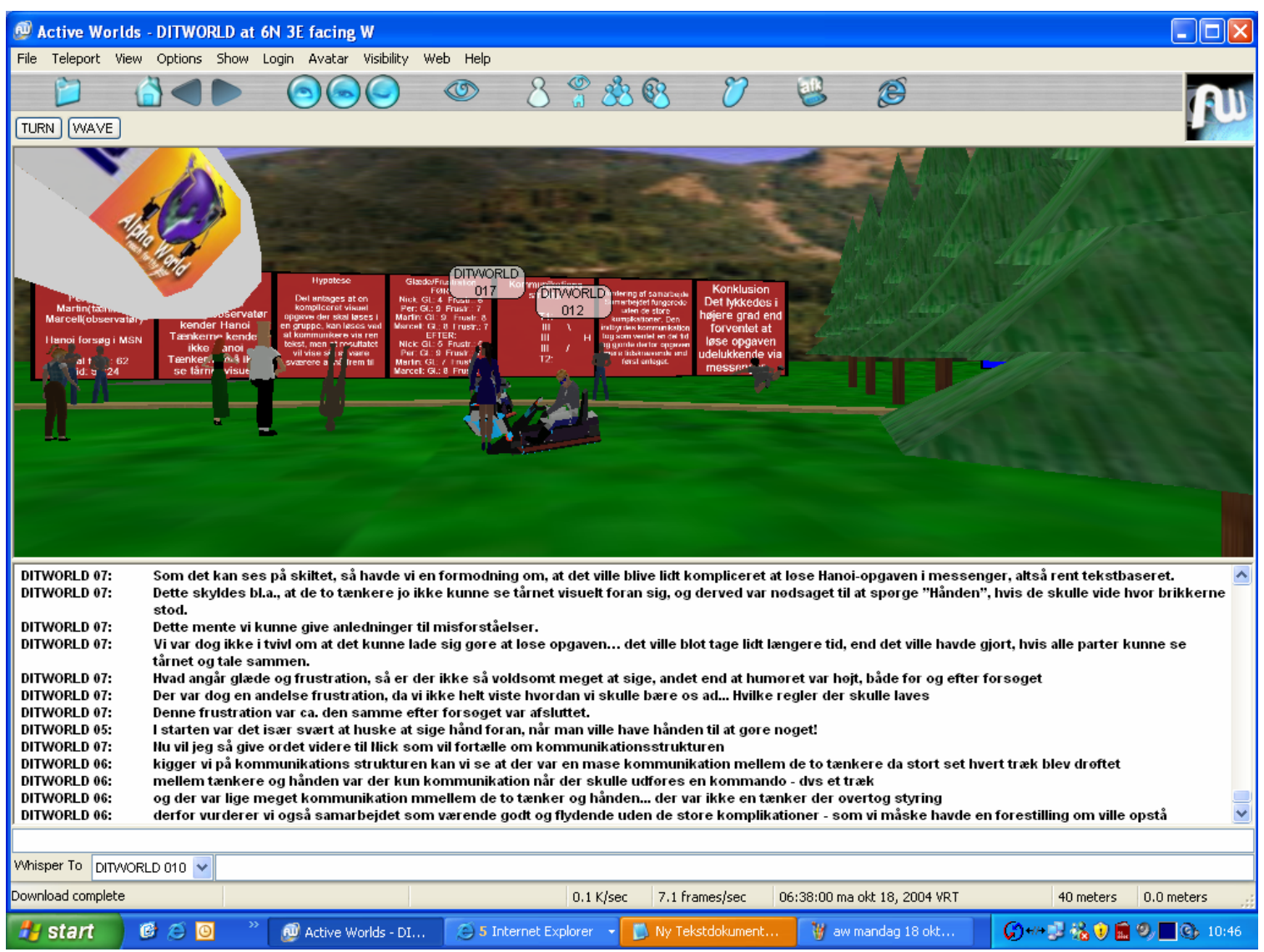

Figure 4. What's going on? The experience of violent online sociability

The airship avatar as well as the motorcycle avatar refers to a transportation reality which is peculiar given that the activity that is going on is a student presentation. The students cannot really speak about how it is to be an airship or be a motorcycle (!), which hinders the alteration of reality; instead one student use his new motorcycle-body to communicate non-verbally (which by the way is what you generally do with a motorcycle) with another student's avatar which in the student-presentation social reality is experienced as a molesting form of sociability. The cultural symbols available in the online social reality refer to a violent sociability that cannot be communicated openly and explicit ${ }^{1}$.

While there is a substantial body of research on avatar violence, which could be discussed in relation to the molestation of the female student, this example also illustrates another point: Participants in networked communication such as the male student may

\footnotetext{
${ }^{1}$ The history of online communities show that from the very beginning molesting has occurred, see http://www.rider.edu/ suler/psycyber/palhistory.html, downloaded September 2005.
} 
have good social skills, even if they in online environments fail to show these skills. Findings from empirical studies of general internet use suggest that users do not become more sociable because they have used the internet, but rather display a higher degree of social connectivity exactly because they as a group are better educated, better off financially and younger that the population on average (Nie, 2001). This line of argument suggest that the young male online molesters in our example may have above average social skills and still end up in a molesting situation in the online environment. However, if we interpret the male student's behavior as a result of inadequate designed technology, we need to be very aware that the other (female) student actually experienced the event very negatively.

Summing up, the examples illustrate communicating about cultural symbols and social reality produce different sociability(ies): Convivial sociability; this is the psychological sense of a social reality that is associated with a communicative coherence in a diversity of ways of being, speaking and perceiving of each other, as in the example with the students accepting the strange new experience of moving collectively online. Superficial sociability; this is the psychological sense of a social reality that is associated with a similarity in appearance and location but not necessarily a shared purpose, as in the example with the three gunslingers at OK corral and the other students. Negative sociability; this is the psychological sense of a social reality that is associated with technology determined, intrusive social acts. This list of different qualities of online sociability is not exhaustive, more might be added and each sociability experience needs to be investigated in detail.

\section{SOLUTIONS AND RECOMMENDATIONS}

The analysis presented here suggests that users are active in designing their own sociability experiences, though they are not successful in all cases. Odd forms of online social interactions emerge such as superficial sociability and convivial sociability, but also as negative sociability. What can we do as designers and researchers to help users' improve their sociability experiences? 
First, we must acknowledge that designers of online communities supply the cultural symbols that through computer mediated communication form and direct participants' psychological sense of the HCI/CSCW enabled social reality - we determine the conditions for the sociability that participants experience. Some conditions are not optimal; it is not easy to address other people if you are a motorcycle or an airship. In the virtual world course, the students should have had the opportunity to choose wise, clever looking, listening avatars within an auditorium of high respect for academia and with means of communication that supported students’ small group presentation.

We as designers should aim at creating cultural symbols that fit the sociability experience that we want our users to have. However, no simple solutions would do. Ways forward would be to write a clear set of rules for how to present in the virtual world such as suggested by the framework for online communities(de Souza \& Preece, 2004), or to moderate the different phases of the community's life, such as suggested by research into portal management (Damsgaard, 2002) and practitioners (Kim, 2000; Powazek, 2002). However, I recommend applying the Social Reality theory of online sociability that has been outlined in this chapter. Application of Social Reality theory make it possible to design and support online sociability with an emphasis on providing rich and relevant cultural symbols and means for communication and feedback about the social reality that is experienced. Furthermore, Social Reality theory of Sociability takes into account psychological essentialism: When a group is perceived as part of social reality, people have a sense of the group's existence being out of their control or manipulation. As designers of networked communication, we must theoretically understand which kind of group we design and support, because the apparent existence of a cluster of people with apparent similarity in members’ goal directed activity, member characteristics such as appearance and disposition, and in physical location, becomes an assumption in peoples' everyday reasoning, and it becomes very hard for the group and group members to alter the group’s characteristics (Kashima, 2004). For example, the three students who designed themselves as Wild West gunslingers should be aware of the consequences of the choice for the production of online sociability and social reality. The groups, which we design, have long lasting consequences for the people who are part of these groups. 
Second, negative sociability must be identified and treated appropriately whenever it occurs. In terms of Social Reality theory, negative sociability may be explained as an effect of lack of communication about cultural symbols and social reality. Hence in order to identify negative sociability, it is necessary to name it as such, when it occurs. For example, in the context of the virtual world university course, any violation of personal space should be addressed by the other students or by the moderator (the teacher) in a (perhaps) similar way as this is done in offline classrooms. This again may require training of the students in social skills by following a standard behavioral program for training of social skills, which will teach them both the environmental social skills needed to perform tasks in educational settings such as listening to other students presentations, and teach them interactional social skills that will help them starting, maintaining and ending all kind of social interactions such as making new friends in a class. These kinds of social skill training have been developed for cooperation in special work settings such as maritime emergencies (Clemmensen, 2001), and for people with special difficulties in the area of everyday social contact (Clemmensen et al., 2000). Such efforts may teach us as online community and networked communication designers to design and plan training in social skills for online users. Furthermore, it is important to add to the reasons for social skills development given in the general literature on social skills training: An individual may not possess an appropriate repertoire of social actions, or do not have had the sufficient opportunity to practice these, or he or she has emotional problems that make it difficult to apply them. As illustrated in the example with negative online sociability, all of these reasons may gain a new actuality when a person moves from offline social interaction to online social interaction. This shift in context presents new application areas and content development for social skills training and support, which may be topics for further research.

Third, superficial sociability is relevant in many situations. As humans, there are many situations where we want to be superficially like others in order to feel at least a small sense of belonging to the group. This experience is valuable online too. In support of online sociability, the gestalt principles of similarity and proximity, which are part of the 
basis for Social Reality theory, may be used to suggest the development of practical solutions. One practical solution for use in virtual world university courses can take form as what I call 'group appearance patterns', i.e. a set of avatars that are designed to be immediately perceived as distinct from other avatars in their appearance, but similar to each other. This will help easy and effective administration and creation of superficial sociability. A second example of a practical solution in the same context is to provide a set of 'listeners' and 'presenters' and 'opponents', etc., i.e. to provide the students with a pre-choice of relevant avatars instead of motorcycles and the like. A third example is to provide pre-defined behavioral settings for different human activities such as student presentations with built-in separate locations for each student group. The main point here is to develop and use positively in online environments our ability to design for similarity in appearance and for proximity in location, which we as humans already are very skilled at in offline situations where we want superficial sociability such as when we dress up for a party or when we try to stand physically close with those we want to belong to.

Fourth, the real positive challenge is to design for convivial sociability. Having a psychological sense of the reality of a social group is strengthened by ongoing mutual verification of collective intentionality: We all the time confirm each other in the group that we aim at a shared target. To the extent that the participants in online communities and networked communication share a common fate, extensive communication allows them to experience a sociability without having to be similar in appearance or proximal in location, i.e. a convivial sociability that according to Social Reality theory emerges as a result of a number of people having extensive communication with and about cultural symbols and extensive communication with and about social reality. These communication processes may result in the creation of new psychological experiences in terms of new cultural models for communication acts in the home (Gourna \& Smoreda, 2003) for social shopping in online stores (Farnham, 2001), for online cantinas (Ducheneaut et al., 2004), and for customer service chat (Andrews \& Haworth, 2002), and many more. 
To further understand the production of convivial online sociability, we may enhance Social Reality theory with two current approaches to the role of communication in the construction of social reality: Articulation Work theory and Sociability Work theory. Articulation work theory is about establishing conventions that are materialized in some artifact (Carla et al., 1999), such as patterns for cooperation in safety critical environments (Stanard \& Wampler, 2005). If it is to produce convivial online sociability, articulation work requires careful consideration of how much of invisible online work that can and should be made visible. On one hand ignoring invisible work may lead to unwanted social consequences (for example that some participants may have to ask all the questions pertaining to where to go, what to do, etc. without getting acknowledged for that work), and on the other hand making the work visible in an artifact also make the work objective and emotionless and object for monitoring by authorities (for example if group creation is supported by formal procedures that is supposed to replace the often emotionally charged processes of group creation) (Star \& Strauss, 1999). Sociability work theory defines sociability work as the work involved in putting on community and charity events (Blackstone, 2004). This kind of work usually has two components: 1) sociability workers must motivate and maintain other volunteer workers by for example putting them selves up as role models, and 2) sociability workers must motivate and maintain donors and event participants, by creating an atmosphere that will make people want to participate (Blackstone, 2004). One important finding from research in sociability work is that this kind of work has costs - personal as well as financial; people that do this kind of work (usually women) may spend many unpaid work hours and face exhaustion when preparing for a community event. Articulation and sociability work theory are both useful additions to Social Reality theory, because Articulation Work theory provides a tool for making invisible work visible, and Sociability Work theory helps analysis of the communication about motivation and social atmosphere, and they both provide the analysis of online sociability with notions of power and hierarchy in social relations. However, these theories do not, as Social Reality theory does, give us access to the users' psychological sense of online social reality. 


\section{FUTURE TRENDS}

One important emerging trend in the literature on online sociability is the possibility for a positive influence of media-life-existence on individuals offline as well as online life (Maloney-Krichmar \& Preece, 2005). Despite negative expectations (Kiesler, 1998) and negative findings (Nie \& Hillygus, 2002) on the impact of internet use on people's sociability, a dedicated use of technology for online sociability might have positive benefits. Benefits stemming from sharing community knowledge (Clemmensen, 2004) and from shared comfort, humor, support (Maloney-Krichmar \& Preece, 2005) are important outcomes of online sociability. The Social Reality theory of Online Sociability should be of value to future research in terms of providing a perspective on online sociability that can encompass and connect to both psychological, sociological and media theories, while keeping a psychological perspective on sociability. For example, it is clear that future studies of the enactment of netiquette may contribute to the Social Reality theory of online Sociability. This research may show how inclusive and open communication processes lead to a non-discriminating online social reality and to the experience of a convivial sociability, given that available cultural symbols (such as appropriate student avatars) are used in the computer mediated communication between participants. An important future research opportunity is how we as designers and researchers support and design for users' experience of being together and having a common fate online without necessarily being similar in appearance or proximal in location, i.e. the investigation of the phenomenological details of the production of convivial sociability.

\section{CONCLUSION}

This chapter review current approaches to online sociability and present a psychological theory, the Social Reality theory, which explains online sociability as the psychological sense of social reality. The chapter uses the results from analyzing a virtual-world university course to illustrate different kinds of online sociability: superficial, convivial, and negative sociability. The chapter gives solutions and recommendations to designers and researchers with a focus on online communities and networked communication. 
Online sociability is today a frequent experience for millions of people, and it will in the years to come be so for many more people across the world. What has been presented here deals in an implicit way with what is known in cognitive anthropology and psychology as cultural models. Obviously the experience of sociability is an outcome of complex and culture-specific structures and processes. In practice this means that planning the sociability of both the products and the dynamic social processes in a knowledge creating context, such as a university course, is only one half of designing for networked communications; the other half is understanding how people with diverse cultural backgrounds actually use these processes and products. To this end, we need to understand diverse cultural practices in terms of cultural models for class room performance, for romance or flirt, for collective movement, etc., both offline and online, in order to design and support for human forms for online sociability.

\section{REFERENCES}

Al-Saggaf, Y., \& Weckert, J. (2004). The effects of participation in online communities on individuals in saudi arabi. SIGCAS Comput. Soc., 34(1), 1.

Andrews, D. C., \& Haworth, K. N. (2002). Online customer service chat: Usability and sociability issues. Journal of Internet Marketing, 2(1).

Avallone, F., Sinangil, H. K., \& Caetano, A. (2005). Convivence in organizations and society (Vol. 12). Milano: Guerini Studio.

Barab, S. A., MaKinster, J. G., Moore, J. A., \& Cunningham, D. J. (2001). Designing and building an online community:The struggle to support sociability in the inquiry learning forum. Etr\&DEducational Technology Research and Development, 49(4), 71-96.

Blackstone, A. M. (2004). Sociability, work, and gender. Equal Opportunities International, 23(3-5), 2945.

Blum-Kulka, S. (1997). Dinner talk - cultural patterns of sociabilit and socialization in family discourse. London: Lawrence Erlbaum.

Campbell, D. T. (1958). Common fate, similarity, and other indicies of the status of aggregates of persons as social entities. Behavioral Science, 3, 14-25.

Carla, S., Gloria, M., \& Dario, G. (1999). Interoperability as a means of articulation work, Proceedings of the international joint conference on Work activities coordination and collaboration. San Francisco, California, United States: ACM Press.

Clemmensen, T. (2001). Tacit social knowledge in safety critical situations (poster). Paper presented at the ECSCW - European Conference on Computer Supported Cooperative Work, Bonn. 
Clemmensen, T. (2004). Community knowledge in an emerging online professional community: The case of sigchi.Dk. Knowledge and Process Management, 11(2), 1-10.

Clemmensen, T., Martinussen, M., \& Skanning, D. (2000). Knowledge and skills in early psychosis: Implications for training (abstract). Paper presented at the 13th International Symposium for the Psychological treatments of Schizophrenia, 5-9 June 2000, The Stavanger Forum Conference Centre, Stavanger, Norway.

Constas, I., \& Papadopoulos, D. (2001). Interface-me: Pursuing sociability through personal devices. Personal Ubiquitous Comput., 5(3), 195-200.

Damsgaard, J. (2002). Managing an internet portal. Communications of the Association for Information Systems, 9, 408-420.

de Souza, C. S., \& Preece, J. (2004). A framework for analyzing and understanding online communities. Interacting with Computers, 16(3), 579.

Ducheneaut, N., Moore, R. J., \& Nickell, E. (2004). Designing for sociability in massively multiplayer games: An examination of the "third places" of swg, Other Players. IT University of Copenhagen, Denmark.

Farnham, S., Zaner, Melora., Cheng, L. (2001). Supporting sociability in a shared browser. Paper presented at the Interact 2001, Tokyo, Japan.

Gourna, C. d., \& Smoreda, Z. (2003). Communication technology and sociability: Between local ties and "global ghetto"? In J. E. Katz (Ed.), Machines that become us: The social context of personal communication technology. New Brunswick: NJ: Transaction Publishers.

Kashima, Y. (2004). Culture, communication and entitativity - a social psychological investigation of social reality. In V. Yzerbyt, C. M. Judd \& O. Corneille (Eds.), The psychology of group perception perceived variability, entitativity and essentialism. NY: Psychology Press.

Kiesler, S. (1998). An internet paradox (panel): A social medium that may undermine sociability, Proceedings of the 1998 ACM conference on Computer supported cooperative work. Seattle, Washington, United States: ACM Press.

Kim, A. J. (2000). Community building on the web - secret strategies for succesful online communities.: Peachbit Press.

Kreijns, K., Kirschner, P. A., \& Jochems, W. (2002). The sociability of computer-supported collaborative learning environments. Educational Technology \& Society, 5(1).

Kreijns, K., Kirschner, P. A., Jochems, W., \& Van Buuren, H. (2004). Determining sociability, social space, and social presence in (a)synchronous collaborative groups. CyberPsychology \& Behavior, $7(2), 155$.

Maloney-Krichmar, D., \& Preece, J. (2005). A multilevel analysis of sociability, usability, and community dynamics in an online health community. ACM Trans. Comput.-Hum. Interact., 12(2), 201-232.

Mantei, M. (1981). The effect of programming team structures on programming tasks. Commun. ACM, 24(3), 106-113. 
Nie, N. H. (2001). Sociability, interpersonal relations, and the internet: Reconciling conflicting findings. The American Behavioral Scientist, 45(3), 420.

Nie, N. H., \& Hillygus, D. S. (2002). The impact of internet use on sociability: Time-diary findings. IT\&Society, 1(1), 1-20.

Powazek, D. M. (2002). Design for community - the art of connecting real people in virtual places: New Riders.

Preece, J. (2000). Online communities - designing usabilty, supporting sociability: Wiley.

Preece, J. (2001). Sociability and usability in online communities: Determining and measuring success. Behaviour \& Information Technology, 20(5), 347.

Rutter, J., \& Smith, G. W. H. (1999). Ritual aspects of cmc sociability. Paper presented at the Esprit i3 Workshop on Ethnographic Studies in Real and Virtual Communities, Queen Margaret University College, Edinburgh.

Ryberg, T., \& Ponti, M. (2004, Monday 5th to Wednesday 7th April). Rethinking virtual space as a place for sociability: Theory and design implications. Paper presented at the Networked Learning Conference 2004, Lancaster University, England, UK.

Simmel, G., \& Hughes, E. C. (1949). The sociology of sociability. American Journal of Sociology, 55(3), 254-261.

Stanard, T., \& Wampler, J. (2005). Work-centered user interface patterns. Paper presented at the INTERACT 2005, Rome.

Star, S. L., \& Strauss, A. (1999). Layers of silence, arenas of voice: The ecology of visible and invisible work. CSCW, 8, 9-30.

Willoughby, T. C. (1972). Staffing the mis function. ACM Comput. Surv., 4(4), 241-259. 